\title{
Pico- to nanosecond pulsed laser-induced forward transfer (LIFT) of silver nanoparticle inks: a comparative study
}

\author{
J. Mikšys ${ }^{1,2}$ (1) G. Arutinov ${ }^{2} \cdot$ G. R. B. E. Römer ${ }^{1}$
}

Received: 29 July 2019 / Accepted: 16 October 2019 / Published online: 7 November 2019

(c) The Author(s) 2019

\begin{abstract}
Silver nanoparticle inks are among the key functional materials used in printed electronics. Depositing it by laser-induced forward transfer remains a challenging task because the non-linear rheological nature of these inks narrows the range of the laser processing parameters. Understanding, therefore, the influence of the laser parameters on the ejection dynamics and deposition quality is of critical importance. The influence of the laser pulse duration from pico- to nanosecond-laser-induced jet dynamics was investigated using time-resolved shadowgraphy imaging. Jet speed and surface area analyses showed that in the lower laser fluence level range, picosecond pulses induce higher surface area ejections which propagate at higher velocities. As the laser fluence levels were increased, the difference in jet velocity and surface area evolutions narrows. Deposition analysis showed a similar behavior with lower transfer thresholds and larger depositions at lower fluence range when picosecond-laser pulses were used.
\end{abstract}

\section{Introduction}

The field of printed electronics has been actively explored for the last couple of decades [1-4]. During this period, a number of printing techniques, such as screen printing [5], inkjet printing [6] and jet printing [7], were optimized for application in display technology [8, 9], RFID tags [10, $11]$, bio-engineering [12] and sensing [13, 14]. While these established techniques have been pushed to their technical limits, there is an industrial need for higher printing resolution and increased printing speed. As well, current printing techniques lack flexibility and/or versatility, e.g. screen printing is characterized by high yield, but it is a mask-based approach $[5,15]$; inkjet printing is digital, but cannot be applied to highly viscous materials [16]; jet printing can operate with viscous materials, but is relatively slow [17]. The increasing needs for flexibility and versatility are driving research to develop a printing technology which is

J. Mikšys

j.miksys@utwente.nl

1 Chair of Laser Processing, Department of Mechanics of Solids, Surfaces and Systems (MS3), Faculty of Engineering Technology, University of Twente, Drienerlolaan 5, 7522NB Enschede, The Netherlands

2 Holst Centre/TNO, High Tech, Campus 31, 5656AE Eindhoven, The Netherlands digital, allowing for printing of micro-deposits with high yield and is applicable to the wide range of materials. Laserbased printing techniques like laser-induced forward transfer (LIFT) provide a potential solution to these demands [18, 19].

Laser-induced forward transfer (LIFT) is a digital, non-contact printing technique, demonstrated for the first time as an approach for printing from solid films in 1986 [20]. This technique employs a laser beam to irradiate a donor material, which is to be transferred onto a receiving substrate as shown in Fig. 1. The donor comprises of a thin film on a carrier substrate. Focusing the laser beam through the carrier (transparent for the wavelength of the laser radiation) onto the carrier-donor interface results in forming a liquid jet, which is propelled towards the target (receiver) substrate. This nozzle-free technique allows to print a wide range of materials, including pure metals [21-23], oxides [24-26], polymers [27, 28], semiconductors [29, 30], ceramics [31], and biomaterials [32-34]. In the field of printed electronics, metal particle-based inks and pastes, such as silver nanoparticle inks, are among the most used donor materials [35-37]. However, these pastes often exhibit non-Newtonian behavior, i.e. a viscosity dependence on the shear rate in the material $[38,39]$. The latter complicates the determination of the range of (laser) parameters, which result in well-controlled ejections and functional depositions [40]. Moreover, these 
Fig. 1 Schematic illustration of the LIFT jetting process. The donor film is a silver nanoparticle ink coated on a transparent (to the laser) carrier substrate and spacers are used to define a printing distance between the receiver substrate and the donor film. Jetting is initiated by focusing the laser beam on the donor-carrier substrate interface

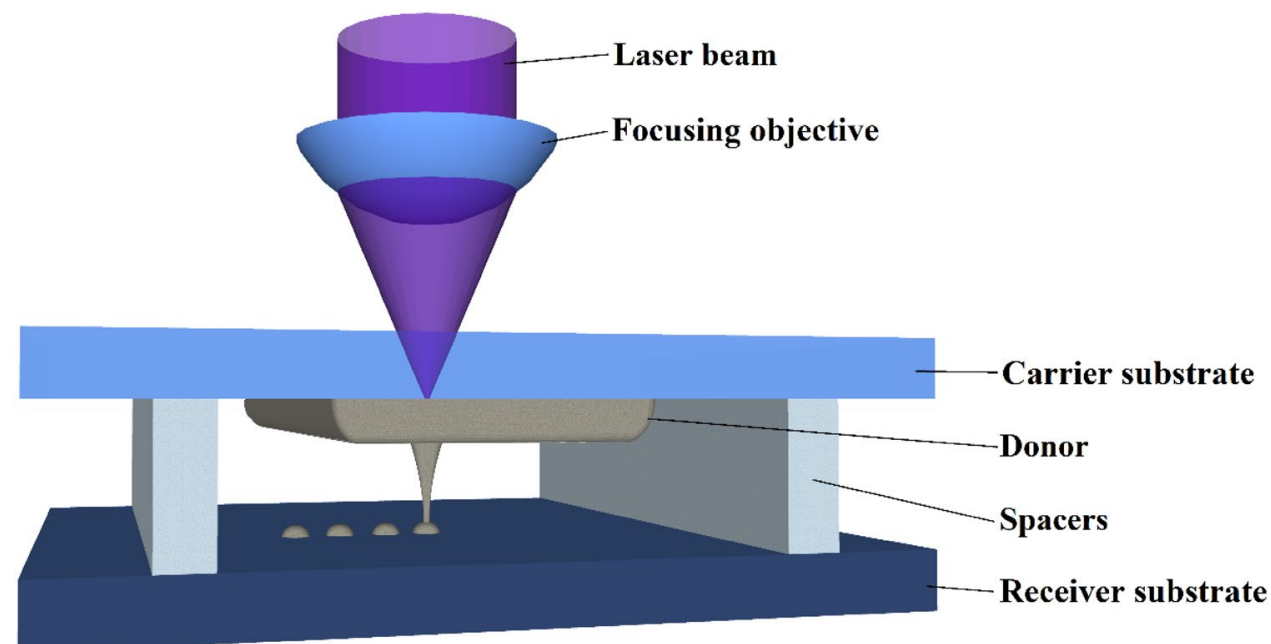

shear thinning inks exhibit a high sensitivity to the laser fluence level used-i.e. a small change of the fluence level can quickly lead to undesired additional droplets (satellites) next to the main droplet, off-angle ejection and/or splashing [41, 42]. Consequently, understanding the effect of the laser parameters on the laser-induced jet dynamics is crucial to print clean depositions and control the morphology of depositions.

Next to the laser fluence, one of the most dominant laser parameters, in any process that involves laser-material interaction, is the duration of the laser pulse. Prior works show that LIFT is commonly executed using laser pulse durations ranging from femtoseconds (fs) to nanoseconds (ns) [42-44]. It was demonstrated that even a CW (continuous wave) laser can be successfully applied to LIFT printing [45]. Prior works studied the LIFT ejection dynamics of gold using laser pulses in the nanosecond and picosecond (ps) range $[22,46]$. It was found that for metal films, the pulse duration played a critical role in the transfer regime. A solid transfer regime was observed for picosecond pulses where nanosecond pulses exhibited melted droplets. Previously, we investigated laser fluence threshold levels at which donor material is ejected from the carrier, when using ps- and nslaser pulses, by analyzing LIFT-printed silver nanoparticle ink depositions [47]. We observed that the fluence threshold is lower when using the ps-laser pulses, compared to ns pulses. This difference was attributed to the additional heat diffusion energy loss to the carrier substrate and the surrounding donor material in case of ns LIFT.

However, no comparative study between ps and ns LIFT was carried with respect to the ejection dynamics-i.e. size and velocity of ejections-of shear thinning, silver nanoparticle based, inks. This paper compares the ejection dynamics of picosecond versus nanosecond LIFT, focusing on the velocity and surface area evolutions in time. The LIFT dynamics study is complemented by the deposition post-analysis, comparing volumes and the diameters of ps and ns LIFT-printed deposits.

\section{Theoretical aspects of ejection dynamics}

Supporting the analysis of the results in Sect. 4, this section gives a brief description of the main stages of the LIFT deposition process. Due to relatively high concentration of silver nanoparticles in the ink, the optical penetration depth of photons of the laser beam into the donor material is dominated by the optical properties of the silver nanoparticles, resulting in an optical penetration depth of just a few hundreds of nanometers. Therefore, the fraction of the incident laser beam which is not reflected, nor scattered, is absorbed by the silver nanoparticles within a thin layer of the donor at the carrier-donor interface, as shown in Fig. 2. The laser energy absorbed by the nanoparticles heats up and evaporates the solvent through heat conduction. As a result, a gas bubble forms, see Fig. 2a. The high pressure $\left(P_{\mathrm{B}}\right)$ in the bubble expands the bubble, "pushing" the donor material away from the carrier substrate, see Fig. 2b. The laser-generated gas bubble is confined by the solid carrier substrate at the top, donor material at the sides and the free surface (donor-air interface) at the bottom. The donor material is more free to move in the direction of the free surface than in the direction of the surrounding liquid. Therefore, the velocity of the bubble surface is higher in the vertical direction than the horizontal direction. Such bubble expansion directionality is highlighted by the black arrows in Fig. 2b. As the bubble expands, $P_{\mathrm{B}}$ decreases and the expansion slows down. When $P_{\mathrm{B}}$ becomes equal or lower (because of the inertia of the liquid in motion) than the pressure outside of the bubble $\left(P_{0}\right)$, the bubble starts to collapse. This induces a flow of donor material from the sides, leading to the formation of a tip at the front of the bubble, presented in Fig. 2c. 


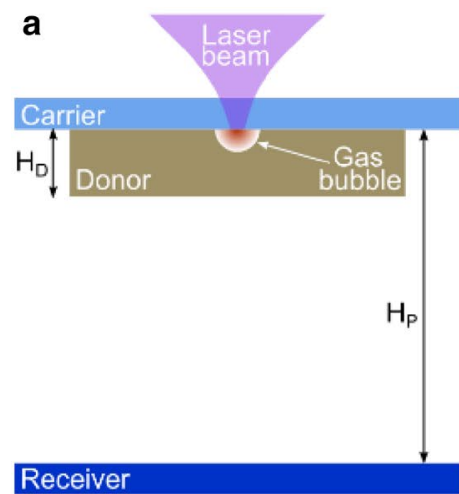

d

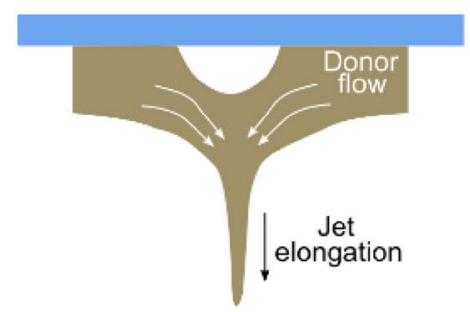

Fig. 2 A schematic diagram of the stages of LIFT, where $H_{D}$ represents the donor layer thickness and $\mathrm{H}_{\mathrm{P}}$ the printing distance. a Highpressure $\left(P_{\mathrm{B}}\right)$ gas bubble generation by the absorbed laser energy; $\mathbf{b}$ bubble expansion due to higher $\mathrm{P}_{\mathrm{B}}$ than the external pressure $\left(P_{0}\right)$; $\mathbf{c}$

As shown in Fig. 2d, while the bubble continues to collapse, the donor material keeps flowing towards the tip and, as a result, the tip evolves into a jet. When the jet reaches the surface of the receiver, it forms a "channel" between the carrier and the receiver substrates. Instead of breaking up, the donor material is being "fed", through the jet, to the receiver, increasing the volume of the deposition. As the material flow slows down, the diameter of the jet reduces, see Fig. 2e. Finally, as the donor material stops flowing, the jet breaks up, lower part of the jet "falls" on the deposition, while the upper part retracts to the donor layer as presented in Fig. $2 \mathrm{f}$.

\section{Materials and methods}

\subsection{Materials}

In this study, a highly viscous silver nanoparticle (NP) ink (Sicrys ${ }^{\text {TM }}$ P75DB-1, PV Nano Cell Ltd., Israel), specifically designed for LIFT printing was used as a donor material. The ink contains spherical silver nanoparticles $(d 50=70 \mathrm{~nm})$ suspended in diethylene glycol monobutyl ether (DGBE) solvent with $75 \mathrm{wt} \%$ metal load [48]. The NP ink is blade coated onto a $20 \times 80 \mathrm{~mm}^{2}, 0.7$-mm-thick

bubble collapse and formation of a tip; $\mathbf{d}$ elongation of the jet; $\mathbf{e}$ jet in contact with the receiver substrate, forming a "channel" between the donor and receiver; $\mathbf{f}$ break-up of the jet

soda-lime glass plate, which was used as a carrier substrate. The distance of the blade above the carrier glass surface $\left(H_{\mathrm{B}}\right)$ was varied to control the thicknesses of donor layers $\left(H_{\mathrm{D}}\right)$. The donor layer thickness and surface roughness were measured using confocal microscopy (Leica DCM 3D by Leica MICROSYSTEMS, Germany) and are summarized in Table 1.

\subsection{Experimental setup}

Two Nd:YAG-based, frequency-tripled laser sources were used. Both lasers emit a wavelength of $355 \mathrm{~nm}$. A

Table 1 Donor layer thickness measurements

\begin{tabular}{lll}
\hline$H_{\mathrm{B}} / \mathrm{um}$ & $H_{\mathrm{D}} / \mathrm{um}$ & $\mathrm{RMS} / \mathrm{um}$ \\
\hline 50 & 35 & 1.8 \\
35 & 20 & 0.9 \\
20 & 13 & 0.3 \\
10 & 7 & 0.5 \\
\hline
\end{tabular}

$H_{B}$ the distance of the blade above the carrier surface, $H_{D}$ measured donor layer thickness, $R M S$ root mean square of the measured donor layer thickness 
Coherent AVIA-355-4500 laser source was used, emitting laser pulses of $t_{\mathrm{p}}=30 \mathrm{~ns}$, while a Coherent Talisker 355-4 produces pulses of $t_{\mathrm{p}}=15 \mathrm{ps}$. The intensity profiles of both laser sources are Gaussian.

Figure 3 shows the time-resolved shadowgraphy imaging setup used to visualize the LIFT ejections. To cover the effective area of the focusing objective, the LIFT laser beam is expanded 1.5 times to the diameter of $5.5 \mathrm{~mm}$ at the fluence level of $1 / \mathrm{e}^{2}$. Then the laser beam is attenuated using the motorized attenuator (Watt Pilot, by Altechna, Lithuania) and focused onto the carrier-donor interface, using the focusing objective (LB1391-A, by Thorlabs $\mathrm{GmbH}$, Germany) of $400 \mathrm{~mm}$ focal length, which produces a $35 \mu \mathrm{m} \pm 3-\mu \mathrm{m}$-diameter laser focal spot.

A piccolo AOT-YVO-1 SP (InnoLas, Germany) emits 80-ps-laser pulses at $532 \mathrm{~nm}$ (green) wavelength and was used to excite fluorescent rhodamine $6 \mathrm{G}$ dye. The latter then emits broad bandwidth light, used to illuminate the LIFT jet. A light homogenizer, composed of a condensing and collimating lens pair (ACL50832U-A, LA1401A, by Thorlabs GmbH, Germany) with the light diffuser (\#47-995, by Edmund Optics, Germany) in between, evenly distributes emitted light and focuses on the plane where LIFT jet is induced. The emitted light, which is not "blocked" by the jet, is captured by the microscope objective and directed to the CCD camera (Prosilica GC1380, Allied Vision, Germany).

A pulse generator is used to trigger single UV pulses of the LIFT laser source, while the delay generator controls the delay time between the latter and the green illumination laser pulse. By scanning the delay, time-resolved evolution of LIFT jet formation can be recorded. The shadowgraphy imaging setup is capable of capturing one image per LIFT event. Therefore, after the image is captured, the carrier substrate is moved, and a next laser pulse is focused on a fresh section of the donor layer position.

\subsection{Methods}

In this work, time-resolved shadowgraphy was used to capture the ejection dynamics of the LIFT. For both the ps and ns experiments, we selected a laser wavelength of $355 \mathrm{~nm}$ since ultraviolet laser radiation is frequently used to LIFT a wide range of materials. The comparison of the LIFT dynamics includes time-resolved analysis of the position of the tip as well as the surface area of the ejections. The temporal length and surface area evolutions were selected to allow for geometrical reconstruction of temporal evolution of the jet. Calculations were carried out assuming axisymmetric shape of the jet. Such an example of a LIFT jet evolution together with the shadowgraphy images captured at the characteristic times is shown in Fig. 4. As the used silver ink is opaque to the wavelength(s) of the light emitted by the rhodamine $6 \mathrm{G}$ dye, we were not able to observe the bubble formation and growth inside the donor layer. Formation of a vapor bubble in LIFT of transparent liquids was analyzed by Duocastella et al. [49].

\section{Results and discussion}

In this section, the comparative results of the LIFT ejection dynamics and deposition dimensions are presented. The velocities as well as the surface area evolutions of ps- and ns-laser-induced jets are compared. Depending on the main LIFT parameters (laser fluence, donor thickness and viscosity of the donor material), the time interval when the jet is in contact with the receiver substrate can range from hundreds
Fig. 3 Schematic illustration of the time-resolved shadowgraphy imaging setup. $B E$ beam expander, $W P$ wave plate, $P$ polarizer, $B D$ beam dump, $M$ mirror, $F O$ focusing objective, $F$ optical fiber, $L H$ light homogenizer, $R D$ rhodamine dye, $C C D$ camera, $M O$ microscope objective

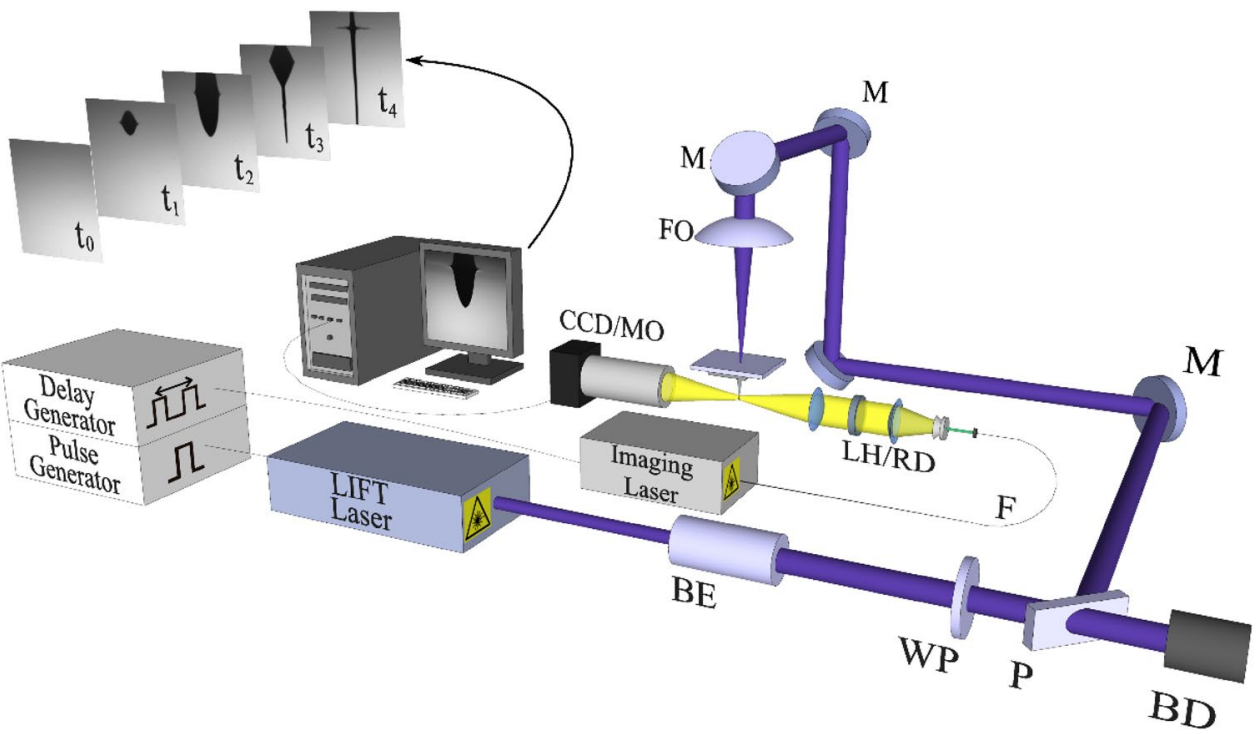




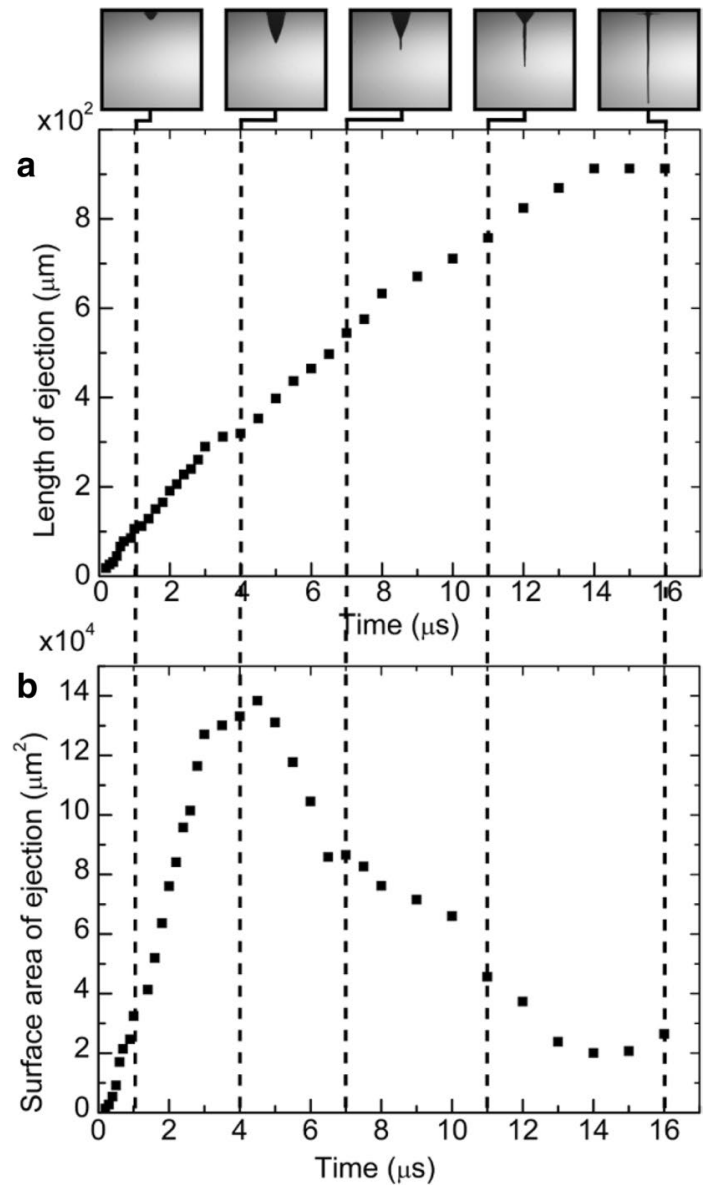

Fig. 4 Typical example of the temporal evolution of the LIFT dynamics a ejection length defined as the distance of the tip from the carrier substrate; $\mathbf{b}$ surface area of the ejected jet. The dotted lines direct to the corresponding shadowgraphy images $\left(1 \times 1 \mathrm{~mm}^{2}\right)$

of microseconds up to milliseconds. Compared to this time range, LIFT jet formation (bubble expansion, bubble collapse and jet elongation) takes place during a relatively short period, and ranges up to tens of microseconds. Since we are only interested in the LIFT ejection dynamics, no receiver substrate was used focusing only on the high-pressure bubble expansion and collapse.

After the time-resolved imaging of the LIFT dynamics, a receiver was placed at $H_{\mathrm{P}}=500 \mu \mathrm{m}$ to transfer deposits and study their dimensions. The volumes and the diameters of the deposits transferred using ps- as well as ns-laser sources are compared.

\subsection{Ejection front velocity analysis}

Figure 5 shows the length of jets, induced by different pulse energies of ps (left) and ns (right) pulses, as a function of time. To make the comparison visually convenient, the horizontal axis corresponding to the ps-laser-induced jets, plotted on the left in this figure, is mirrored with respect to the horizontal axis of the ns-laser-induced jets, on the right of the graph. Bubble front velocity was extracted from the measured jet length by applying the linear fit of the jet length data. This method was previously applied in the study of high-viscosity silver nanoparticle pastes $[42,50]$. In our study, the change of the ejection front speed at the point where bubble reaches the collapsing stage (at about $4 \mu$ in Fig. 5) is evident. Therefore, the linear fit was applied up to this point.

Figure 5 shows that ps-laser-induced bubbles develop faster than those induced by the nanosecond pulses. This difference is significant at low pulse energies $(1-5 \mu \mathrm{J})$, but negligible for jets induced by pulse energies over $8 \mu \mathrm{J}$. This is attributed to the combination of two effects-heat diffusion during the absorption of the laser pulse and the shear thinning effect of the silver ink, that is, during the bubble expansion, the governing force originates from the difference between the (atmospheric) pressure outside the donor material $P_{0}$ and the gas pressure inside the bubble $P_{\mathrm{H}}$. The absorption volume-i.e. the volume where the laser energy is absorbed-is defined by the diameter of the laser beam focused on the donor surface and the optical penetration depth of the laser radiation, which depends on the material and the laser wavelength.

For beams with a similar diameter, the energy absorbed within the volume is identical for ps- and ns-laser pulses. However, the volume in the donor where the material is evaporated exceeds the absorption volume, because the absorbed laser energy diffuses into the ink. The length over which material is evaporated can be estimated from the thermal diffusion distance $D$, which reads [51]

$D=\sqrt{\pi \alpha t_{\mathrm{p}}}$,

where $t_{\mathrm{p}}$ is the laser pulse duration and $\alpha$ is thermal diffusivity of the donor material. Since the donor material used in the experiments was fixed, the only variable affecting the thermal diffusion length is laser pulse duration. By estimating the ratio $D_{30 \mathrm{~ns}} / D_{15 \mathrm{ps}}$, it can be concluded that, after $t_{\mathrm{p}}=30 \mathrm{~ns}$, the thermal diffusion distance is about 45 times larger than the thermal diffusion distance for $t_{\mathrm{p}}=15 \mathrm{ps}$. Consequently, with the same pulse energy, beam diameter and wavelength, the picosecond-laser pulse will generate a more geometrically confined bubble with a higher initial internal pressure than a nanosecond pulse. This explains the difference of the ejection velocities between ns- and ps-induced jets, at low-energy pulses. The increase of the laser pulse energy results in a higher pressure of the initial bubble, inducing higher shears inside the donor material. Since the used silver nanoparticle ink exhibits the shear thinning effect, higher shears will result in a drop of viscosity of the donor material. As the viscosity drops, the donor material becomes less resistant to deformation (motion of 


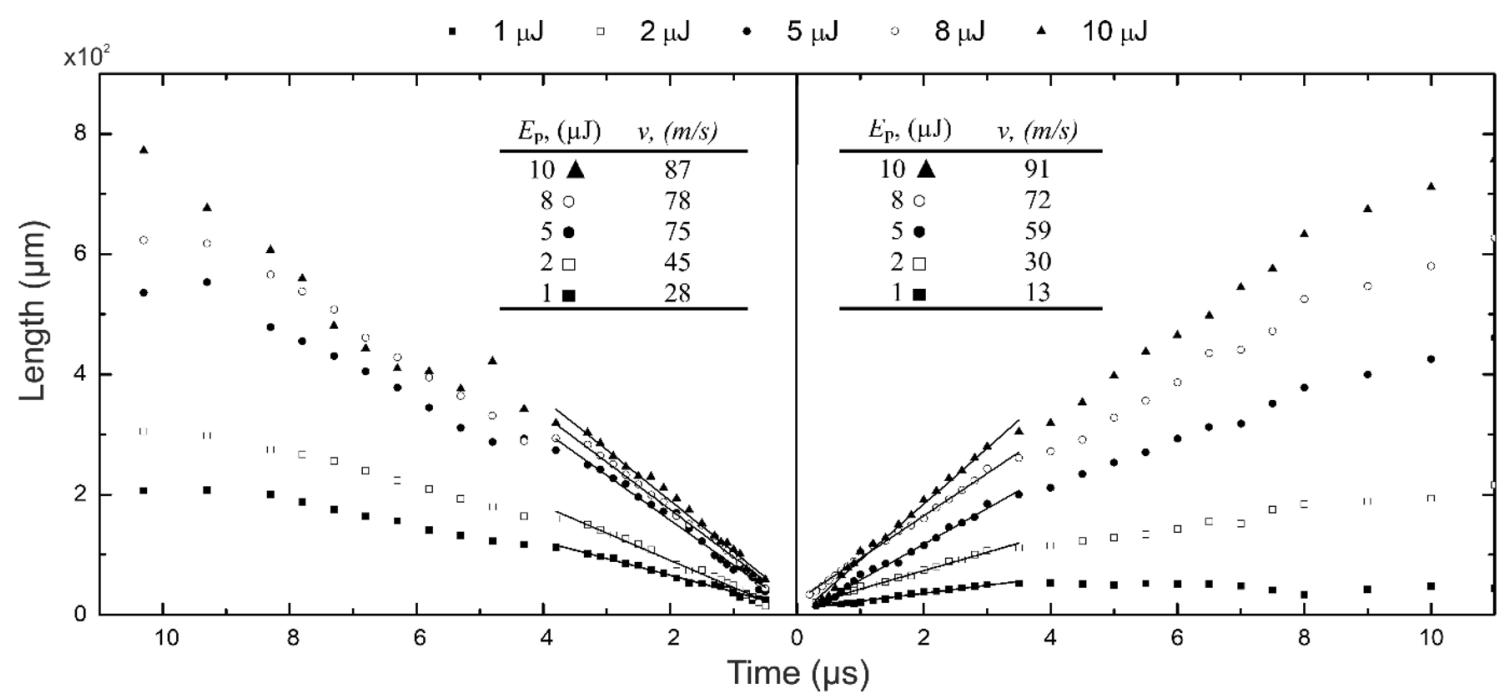

Fig. 5 Jet lengths as a function of time, using different laser pulse energies. Right: jet length induced by 30-ns-laser pulses. Left: jet length induced by 15 -ps-laser pulses. Note that the horizontal axes (time) of these graph are mirrored to allow comparison of the phe-

the liquid). Therefore, the difference in thermal diffusion distance between ns- and ps-LIFT diminishes with higher energy laser pulses.

In printed electronics, the size of deposited droplets (depositions) and printing distance (between carrier and receiving substrate) are of critical importance. More precisely, the latter is described as a distance between the bottom of the donor layer and the surface of the receiver substrate. Both deposition size and possible printing distance range are controlled through the laser fluence, laser beam diameter at the carrier-donor interface and the thickness of the donor layer. To investigate the role of these parameters, a set of LIFT experiments was performed. They were repeated using four different thicknesses of donor layers, ranging from $7 \mu \mathrm{m}$ to $35 \mu \mathrm{m}$. Each of the four layers was exposed to three different laser spot diameters, namely $35 \mu \mathrm{m}, 80 \mu \mathrm{m}, 160 \mu \mathrm{m}$, and five different pulse energies per spot diameter. To normalize the data, the latter two parameters were combined into laser fluence $F_{\mathrm{L}}=8 E_{\mathrm{P}} / \pi d^{2}$, where $E_{\mathrm{P}}$ denotes the laser pulse energy and $d$ is the diameter $\left(1 / \mathrm{e}^{2}\right)$ of the laser spot. The bubble front velocities depicted in Fig. 5 were calculated from the linear fitting of length graphs. Figure 6 shows the bubble front velocities ratio $v_{\mathrm{ps}} / v_{\mathrm{ns}}$ of the ps jet velocity $v_{\mathrm{ps}}$ versus the ns jet velocity $v_{\mathrm{ns}}$, as function of the laser fluence for four different thickness donor layers. For laser fluences less than about $0.5 \mathrm{~J} / \mathrm{cm}^{2}$, the bubble front velocity ratio is larger than 1. In this laser fluence regime, the ps-laserinduced bubble front is moving two to eight times faster than bubbles induced by ns-laser pulses, see Fig. 7a. When the laser fluence, however, exceeds $0.5 \mathrm{~J} / \mathrm{cm}^{2}$, the velocity ratio does go to 1 and the front velocity of the bubbles induced by nomena in time. Linear curve fitting of the jet length was applied over a time range of $0-4 \mu \mathrm{s} . R^{2}$ values of the curve fits are higher than $97 \%$. The reproducibility of every data point in the graph is over $95 \%$. The inserted tables indicate the front velocity of the bubble

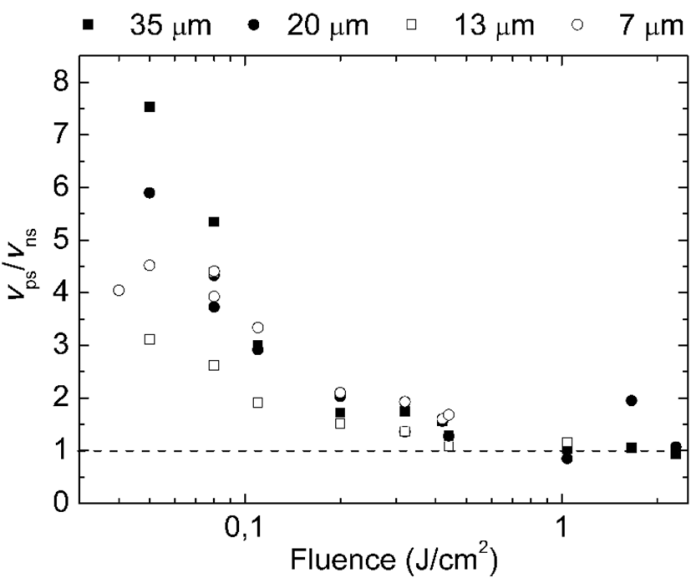

Fig. 6 Ratio $v_{\mathrm{ps}} / v_{\mathrm{ns}}$ of the ps jet velocity $v_{\mathrm{ps}}$ and the ns jet velocity $v_{\mathrm{ns}}$ as a function of the laser fluence for four different thickness donor layers

the ps- and ns-laser pulses equalize. An example of such jets induced by high laser fluence travelling with similar speeds for ps and ns pulses is shown in Fig. 7b.

\subsection{Ejection surface area analysis}

The ejection length and velocity analyses, shown in Figs. 5 and 6, only provide information about the front of the ejection. To obtain a comprehensive description of the LIFT jet dynamics and compare jets induced by the two different pulse durations, the evolution of the surface area of the bubble was derived from the shadowgraphy images. The 
Fig. 7 Shadowgraphy images of LIFT. a Low-fluence regime showing different jet dynamics due to ps and ns pulses; $\mathbf{b}$ similar jet dynamics induced by ns and ps pulses, when high-fluence pulses are used. The size of every shadowgraphy image is $400 \times 1200 \mu \mathrm{m}^{2}$
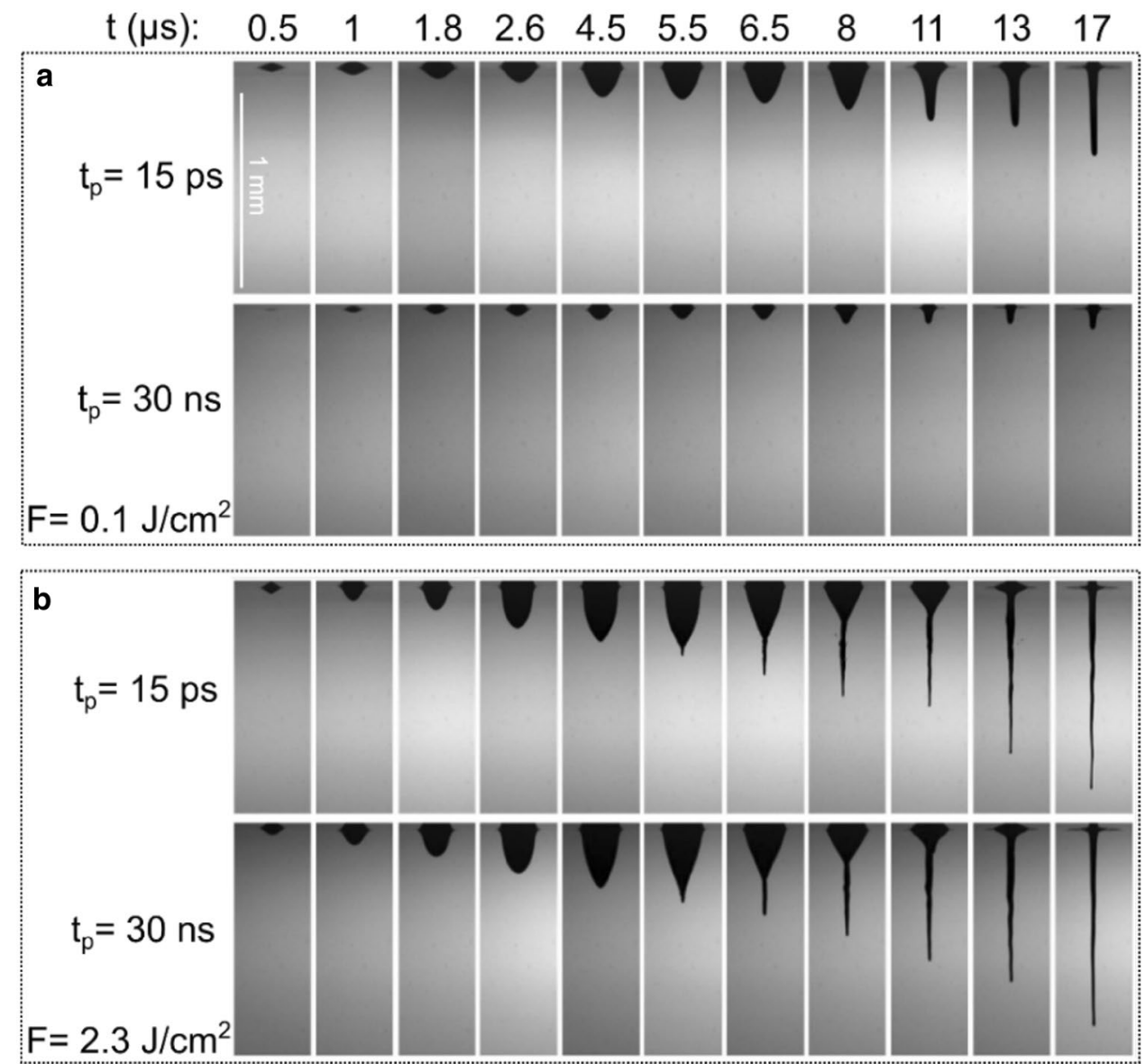

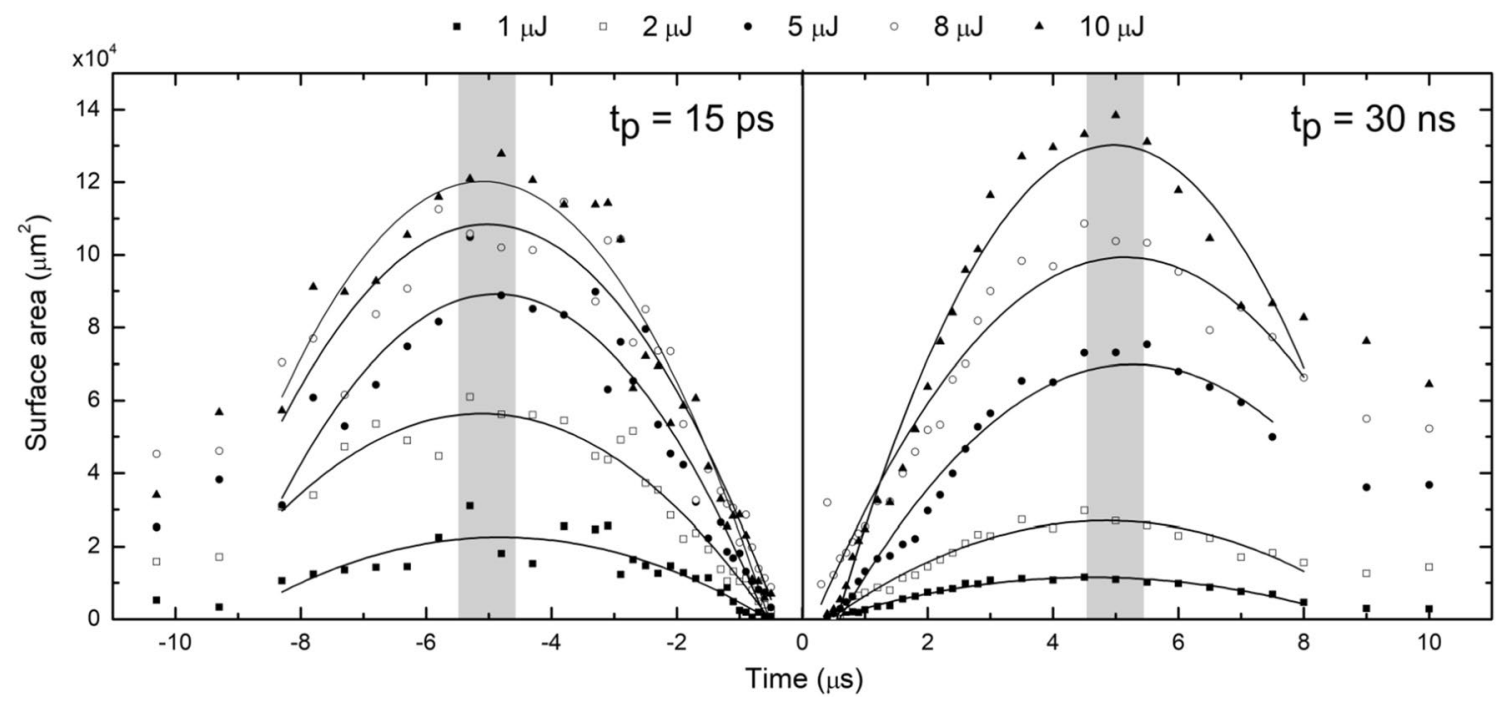

Fig. 8 Surface area of LIFT ejections as a function of time. Secondorder polynomial fitting of the ejection surface area was applied over a time range of $0-8 \mu \mathrm{s}$. $R^{2}$ values of the curve fits are higher than
$91 \%$. The reproducibility of every data point in the graph is over $95 \%$. Gray area marks the time instance of the maximum surface area of the ejection 
surface area at each instance was calculated from the $2 \mathrm{D}$ time-resolved shadowgraphy images, assuming axial symmetry of the jets. The laser-induced jet surface area dependence as a function of time is shown in Fig. 8.

For visual convenience, the horizontal axis representing the ps-laser-induced ejections on the left side of the graph is mirrored with the respect to the ns-laser data on the right. It is observed from Fig. 8 that, in the case of ps-LIFT, the spread in the data is larger than in the case of ns-LIFT. This is attributed to limitations in the experimental setup. It was found empirically that, during the laser-induced bubble growth phase and the beginning of the collapse phase, the temporal surface area evolution follows a second-order polynomial function. However, as the bubble collapse slows down and the bubble turns into a jet (around $8 \mu$ s in Fig. 8), the jet surface area evolution deviates from this quadratic function. Therefore, second-order polynomial fitting was applied up to this time instance.

From Fig. 8 it is evident that when lower pulse energies are applied, the maximum surface area is higher in case of ps-laser pulses, than in case of ns pulses. This means that in the lower pulse energy range, a picosecond pulse is able to eject more material than a ns pulse. However, as higher pulse energies are applied, i.e. higher than $5 \mu \mathrm{J}$, the difference of the maximum surface area of the ejection induced by picosecond and nanosecond pulses becomes negligible. This result coincides with that presented in 4.1 and could also be reasoned by the heat diffusion analysis and the shear thinning of silver nanoparticle ink.

Furthermore, Fig. 8 suggests that the time instance at which the laser-induced jet reaches the maximum surface area does not depend on the pulse energy. This observation was confirmed by plotting similar surface area evolutions for different laser spot diameters and different thickness of donor layers. Figure 9 shows the time instance at which the maximum jet surface area is reached $t_{\mathrm{Smax}}$, as a function of

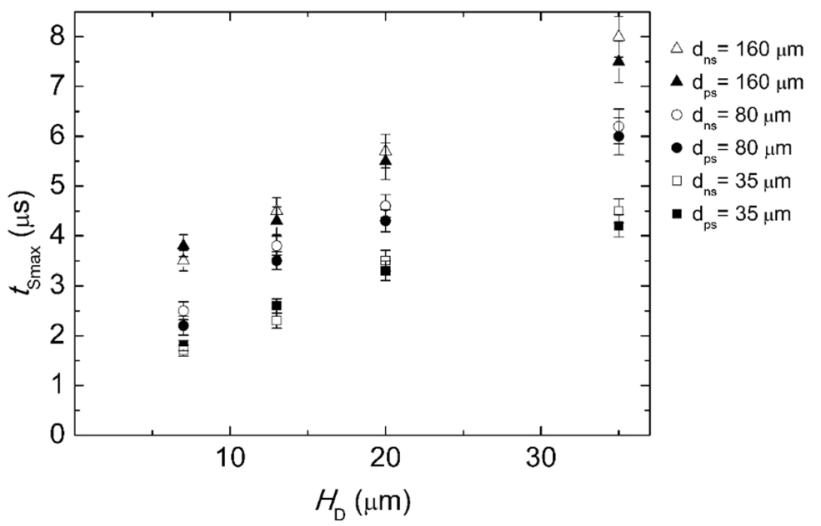

Fig. 9 Time instance at which the maximum jet surface area is reached, as a function of the donor layer thickness for different diameters of the laser spot the donor layer thickness for different diameters of the laser spot. From this graph, we concluded that $t_{\mathrm{Smax}}$ increases with increasing donor layer thickness. A similar dependence was found, when analyzing the time instance at which the maximum surface area is reached for a specific laser spot diameter. The larger the laser spot at the carrier-donor interface, the more the material to be displaced. The same tendency holds for the increase of the donor layer thickness. This means that the time instance of the maximum surface area is a function of the donor layer. Surprisingly $t_{\mathrm{S} \max }$ is independent of the ps to ns pulse length. Therefore, this observation will be addressed in future works.

\subsection{Deposition morphology, diameter and volume}

This section compares the diameter and volume of silver nanoparticle ink depositions printed using ps- and ns-laser pulses. The deposits were printed onto the receiver from two donor layers having different thicknesses $H_{\mathrm{D}}$, namely $7 \mu \mathrm{m}$ and $35 \mu \mathrm{m}$. A printing distance $H_{\mathrm{P}}$ of $500 \mu \mathrm{m}$ was selected. A laser spot diameter of $60 \mu \mathrm{m}$ was used, allowing a fluence level scan up to $0.8 \mathrm{~J} / \mathrm{cm}^{2}$. For statistical analysis of the reproducibility, LIFT deposition experiments were repeated ten times for each set of the parameter combination. Only cases with all ten successful depositions were analyzed. The lower laser fluence level cases, where not all jets reached the receiver substrate, were disregarded.

Figure 10 shows confocal microscopy image of typical deposits induced by ns-LIFT (left images) and ps-LIFT (right images). As is observed from this figure, the size of the deposits of ps-LIFT are larger than that of ns-LIFT deposits, when low $\left(0.2 \mathrm{~J} / \mathrm{cm}^{2}\right)$ laser fluence level pulses are used. This is the case for both $35 \mu \mathrm{m}$ (Fig. 10a, b) and $7 \mu \mathrm{m}$ (Fig. 10e, f) donor layer thicknesses. However, when higher laser fluence pulses (of $0.6 \mathrm{~J} / \mathrm{cm}^{2}$ ) are used for $35 \mu \mathrm{m}$ donor layer thickness, there is no significant difference in the shape and size of ps-LIFT and ns-LIFT depositions ejected, see Fig. 10c, d. Nevertheless, when applying the same fluence levels to a donor layer with a thickness of $7 \mu \mathrm{m}$, debris is observed next to the main (large) deposition, both in psLIFT and ns-LIFT cases, see Fig. 10g, h.

The deposition volume (a,b) and diameter (c,d) as a function of the laser fluence for different donor layer thickness $H_{\mathrm{D}}$ and two pulse durations, printed using printing distance $H_{\mathrm{P}}=500 \mu \mathrm{m}$ are presented in Fig. 11. When a donor layer with a thickness of $H_{\mathrm{D}}=35 \mu \mathrm{m}$ was used, only clean depositions were observed, even up to the maximum used fluence level of $0.8 \mathrm{~cm} / \mathrm{J}^{2}$.

Compared to a donor layer thickness of $35 \mu \mathrm{m}$, when a thinner donor layer (here $H_{\mathrm{D}}=7 \mu \mathrm{m}$ ) is used, the range of the laser fluence levels, where clean depositions are observed, is smaller, see Fig. 11. There are three main reasons, why thinner donor layers exhibit unclean depositions. First, if jet 


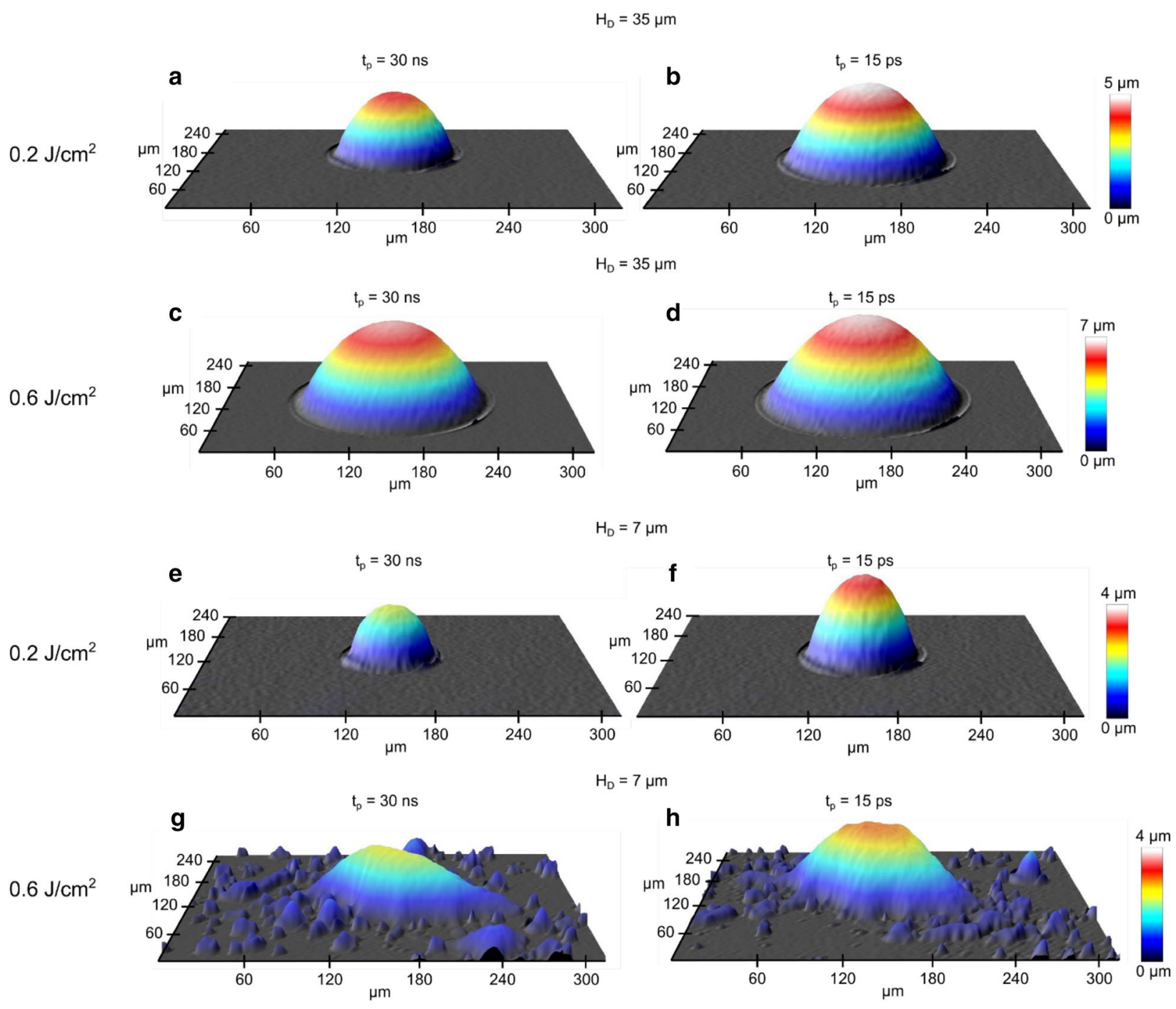

Fig. 10 Isometric confocal microscope images of typical ns-LIFT depositions (left images) and ps-LIFT depositions (right images) for varying laser fluence $(F)$ and donor layer thickness $\left(H_{\mathrm{D}}\right)$ at a

is propagating at a velocity higher than the thin(er) donor layer can "feed", the jet thickness reduces too rapidly, breaking up before reaching the receiver substrate. As a result, a droplet (or multiple droplets) of the donor material falls on the receiver substrate. Second, if the laser fluence level is further increased, the jet becomes turbulent, resulting in even more debris. Turbulent jetting regime is a known phenomenon and was reported by Boutopoulos et al. [42]. Last, further increasing the laser fluence level results in a pressure $P_{\mathrm{B}}$ rupturing the bubble, spraying many small droplets onto the receiver substrate.

Figure 10 shows that the fluence threshold at which successful depositions occur is lower in case of ps-LIFT than for ns-LIFT. In addition, for ps-LIFT, the diameter and volume of depositions at low-fluence levels are lager. However, when the fixed printing distance $H_{\mathrm{P}}=500 \mu \mathrm{m}$. a, b $F=0.2 \mathrm{~J} / \mathrm{cm}^{2}, H_{\mathrm{D}}=35 \mu \mathrm{m}$; c, d $F=0.6 \mathrm{~J} / \mathrm{cm}^{2}, H_{\mathrm{D}}=35 \mu \mathrm{m} ; \mathbf{e}, \mathbf{f} F=0.2 \mathrm{~J} / \mathrm{cm}^{2}, H_{\mathrm{D}}=7 \mu \mathrm{m} ; \mathbf{g}, \mathbf{f}$ $F=0.6 \mathrm{~J} / \mathrm{cm}^{2}, H_{\mathrm{D}}=7 \mu \mathrm{m}$

laser fluence level reaches $0.5 \mathrm{~J} / \mathrm{cm}^{2}$, differences in deposition dimensions between ps-LIFT and ns-LIFT become negligible, see Fig. 11. This $0.5 \mathrm{~J} / \mathrm{cm}^{2}$ fluence level matches with the one where the difference between the speed of the ps-induced and ns-induced jets diminishes as shown in Fig. 6. For thinner donor layer $(7 \mu \mathrm{m})$, however, the effect could not be seen because higher fluence levels induced satellite droplets, turbulent jets and material splashing, resulting in debris on the receiver. 
Fig. 11 Volume (a, b) and diameter (c, d) of silver nanoparticle ink depositions, as a function of the laser fluence for different donor layer thickness $H_{\mathrm{D}}$ and two pulse durations. a, c $H_{\mathrm{D}}=35 \mu \mathrm{m} ; \mathbf{b}, \mathbf{d} H_{\mathrm{D}}=7 \mu \mathrm{m}$. Printing distance $H_{\mathrm{P}}=500 \mu \mathrm{m}$. Standard deviation of every data point is lower than $3 \%$
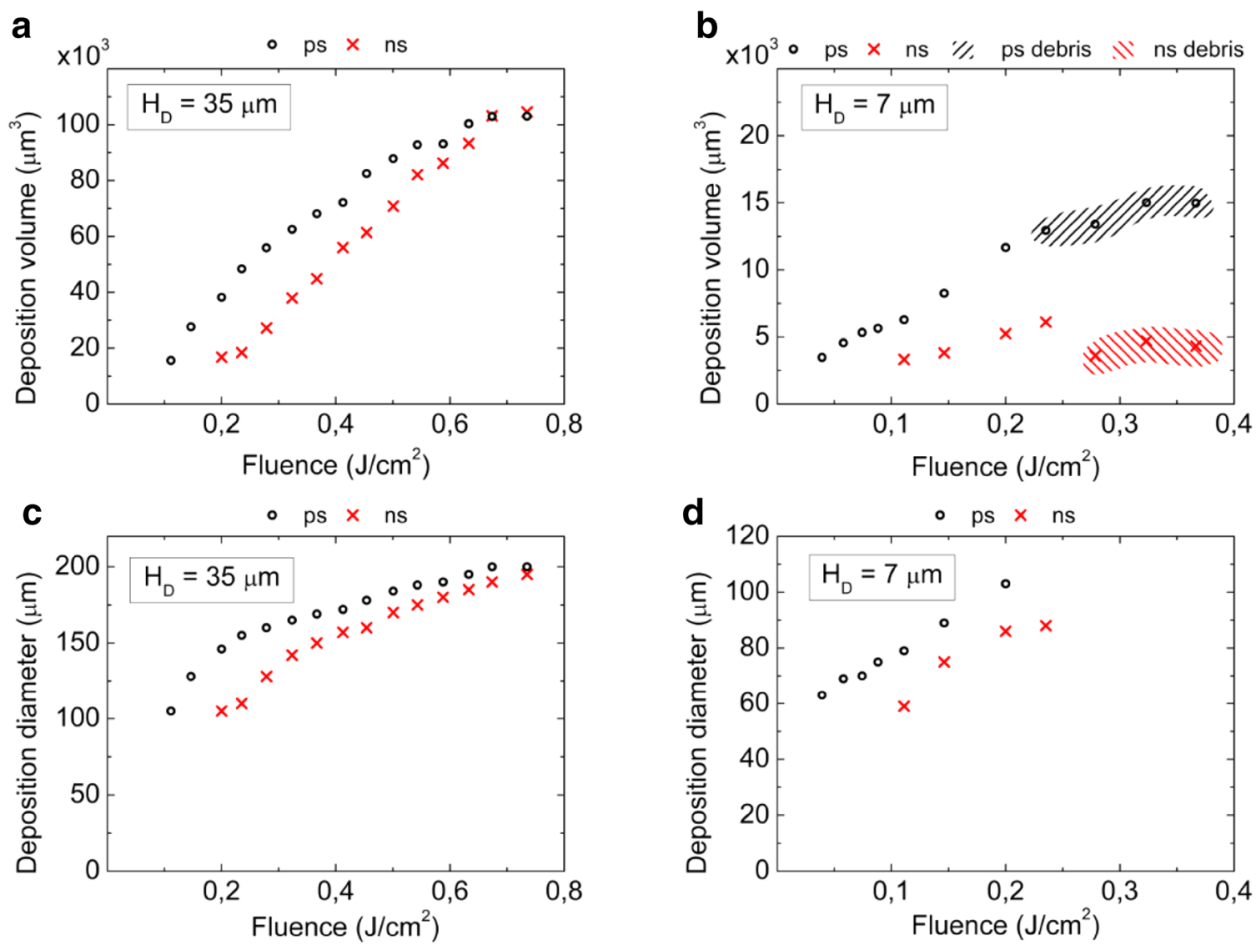

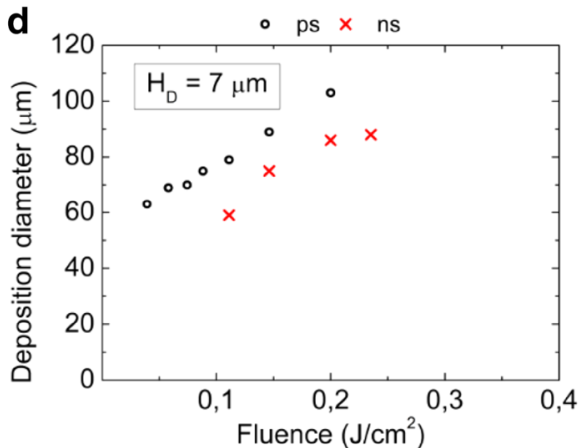

\section{Conclusions}

In this work, we used time-resolved shadowgraphy imaging to compare the dynamics of silver nanoparticle ink ejections, induced by ps- and ns-laser pulses. We found that at relatively low-fluence levels $\left(<0.5 \mathrm{~J} / \mathrm{cm}^{2}\right)$, the velocity of jets induced by ps-LIFT is higher than those induced by nsLIFT. Also, the maximum surface area of LIFT ejections induced by ns pulses is smaller than the ones induced by ps-laser pulses. This was attributed to the lower thermal diffusion length in case of ps pulse, leading to a more confined initial bubble and higher internal pressure. In the case of higher fluence levels $\left(>0.5 \mathrm{~J} / \mathrm{cm}^{2}\right)$, the difference in jet velocity and surface area diminished. This observation coincides with the reduced impact of thermal diffusion on the initial bubble pressure and the drop in viscosity of the silver nanoparticle ink at higher fluence levels. Analysis of the deposition diameter and volume showed a clear correlation with the time-resolved dynamics analysis, where ps-LIFT jets showed lower deposition threshold and larger depositions at lower laser fluence levels compared to ns-LIFT.

With this shadowgraphy imaging study, we provide new insights into understanding the role of the laser pulse duration from ps to ns on the LIFT process of silver nanoparticle ink. Whereas pulse duration is a major parameter in the laser-material interaction for solid film-based LIFT, in the case of highly viscous inks, where a gas bubble is generated, pulse duration does not play a significant role. These results confirm that deposits transferred by picosecond-laser system can also be obtained using more cost-effective nanosecondlaser sources.

Acknowledgements This work is part of the research project, titled "High-resolution deposition of high-viscosity materials using Laser Induced Forward Transfer" (Hi-LIFT, Project Number 14641), which is (partly) financed by the Dutch Research Council (NWO). The authors would like to thank Merijn Giesbers and Edsger Smits for inspiring discussions.

Open Access This article is distributed under the terms of the Creative Commons Attribution 4.0 International License (http://creativeco mmons.org/licenses/by/4.0/), which permits unrestricted use, distribution, and reproduction in any medium, provided you give appropriate credit to the original author(s) and the source, provide a link to the Creative Commons license, and indicate if changes were made.

\section{References}

1. S. Khan, L. Lorenzelli, and R. S. Dahiya, IEEE Sens. J. 15, 3164 (2015).

2. C. Ru, J. Luo, S. Xie, and Y. Sun, J. Micromech. Microeng. 24 , 053001 (2014).

3. V. Subramanian, J. B. Chang, A. De La Fuente Vornbrock, D. C. Huang, L. Jagannathan, F. Liao, B. Mattis, S. Molesa, D. R. Redinger, D. Soltman, S. K. Volkman, and Q. Zhang, ESSCIRC 2008 - Proc. 34th Eur. Solid-State Circuits Conf. 17 (2008).

4. W. Zeng, L. Shu, Q. Li, S. Chen, F. Wang, X.M. Tao, Adv. Mater. 26, 5310 (2014)

5. D.E. Riemer, Microelectron. Int. An Int. J. 6, 8 (1989)

6. M. Singh, H.M. Haverinen, P. Dhagat, G.E. Jabbour, Adv. Mater. 22, 673 (2010) 
7. S. Sanaur, A. Whalley, B. Alameddine, M. Carnes, and C. Nuckolls, Org. Electron. Physics, Mater. Appl. 7:423 (2006).

8. M. Bale, J.C. Carter, C.J. Creighton, H.J. Gregory, P.H. Lyon, P. $\mathrm{Ng}$, L. Webb, A. Wehrum, J. Soc. Inf. Disp. 14, 453 (2006)

9. H.S. Koo, M. Chen, P.C. Pan, L.T. Chou, F.M. Wu, S.J. Chang, T. Kawai, Displays 27, 124 (2006)

10. L. Yang, A. Rida, R. Vyas, M.M. Tentzeris, I.E.E.E. Trans, Microw. Theory Tech. 55, 2894 (2007)

11. A. Blayo and B. Pineaux, 27 (2006).

12. T. Boland, T. Xu, B. Damon, X. Cui, Biotechnol. J. 1, 910 (2006)

13. S. M. Zakir Hossain, R. E. Luckham, A. M. Smith, J. M. Lebert, L. M. Davies, R. H. Pelton, C. D. M. Filipe, and J. D. Brennan, Anal. Chem. 81, 5474 (2009).

14. Q. Qi, T. Zhang, Q. Yu, R. Wang, Y. Zeng, L. Liu, H. Yang, Sensors Actuators B Chem. 133, 638 (2008)

15. F.C. Krebs, J. Fyenbo, M. Jørgensen, J. Mater. Chem. 20, 8994 (2010)

16. N. Reis, B. Derby, MRS Bull. 28, 815 (2003)

17. X.B. Chen, H. Ke, I.E.E.E. Trans, Electron. Packag. Manuf. 29, 75 (2006)

18. M. Morales, D. Munoz-Martin, A. Marquez, S. Lauzurica, and C. Molpeceres, Laser-Induced Forward Transfer Techniques and Applications, Second Edn (Elsevier Ltd., 2017).

19. A. Piqué and P. Serra, Laser Printing of Functional Materials: 3D Microfabrication, Electronics and Biomedicine (2018).

20. J. Bohandy, B.F. Kim, F.J. Adrian, J. Appl. Phys. 60, 1538 (1986)

21. M. Zenou, A. Sa'Ar, and Z. Kotler, Small 11, 4082 (2015).

22. R. Pohl, C. W. Visser, G. W. Römer, D. Lohse, C. Sun, and B. Huis in 'T Veld, Phys. Rev. Appl. 3, 1 (2015).

23. C. Unger, J. Koch, L. Overmeyer, B.N. Chichkov, Opt. Express 20, 24864 (2012)

24. E. Fogarassy, C. Fuchs, F. Kerherve, G. Hauchecorne, J. Perriere, J. Mater. Res. 4, 1082 (1989)

25. I. Zergioti, S. Mailis, N.A. Vainos, P. Papakonstantinou, C. Kalpouzos, C.P. Grigoropoulos, C. Fotakis, Appl. Phys. A Mater. Sci. Process. 66, 579 (1998)

26. S. Rung, A. Christiansen, R. Hellmann, Appl. Surf. Sci. 305, 347 (2014)

27. G.B. Blanchet, Macromolecules 28, 4603 (1995)

28. R. Fardel, M. Nagel, F. Nüesch, T. Lippert, A. Wokaun, Appl. Surf. Sci. 255, 5430 (2009)

29. L. Rapp, A.K. Diallo, S. Nénon, A.P. Alloncle, C. Videlot-Ackermann, F. Fages, M. Nagel, T. Lippert, P. Delaporte, Thin Solid Films 520, 3043 (2012)

30. A. Narazaki, T. Sato, R. Kurosaki, Y. Kawaguchi, H. Niino, Appl. Phys. Express 1, 0570011 (2008)

31. K. S. Kaur, R. Fardel, T. C. May-Smith, M. Nagel, D. P. Banks, C. Grivas, T. Lippert, and R. W. Eason, J. Appl. Phys. 105, (2009).
32. F. Guillemot, A. Souquet, S. Catros, B. Guillotin, J. Lopez, M. Faucon, B. Pippenger, R. Bareille, M. Rémy, S. Bellance, P. Chabassier, J.C. Fricain, J. Amédée, Acta Biomater. 6, 2494 (2010)

33. B. Guillotin, F. Guillemot, Trends Biotechnol. 29, 183 (2011)

34. L. Koch, S. Kuhn, H. Sorg, M. Gruene, S. Schlie, R. Gaebel, B. Polchow, K. Reimers, S. Stoelting, N. Ma, P.M. Vogt, G. Steinhoff, B. Chichkov, Tissue Eng. Part C Methods 16, 847 (2009)

35. J. Perelaer, P.J. Smith, D. Mager, D. Soltman, S.K. Volkman, V. Subramanian, J.G. Korvink, U.S. Schubert, J. Mater. Chem. 20, $8446(2010)$

36. A. Kamyshny, S. Magdassi, Small 10, 3515 (2014)

37. A. Kamyshny, J. Steinke, and S. Magdassi, 1 (2011).

38. E. Tamjid, B.H. Guenther, Powder Technol. 197, 49 (2010)

39. I.M. Krieger, T.J. Dougherty, Trans. Soc. Rheol. 3, 137 (1959)

40. A. Kalaitzis, M. Makrygianni, I. Theodorakos, A. Hatziapostolou, S. Melamed, A. Kabla, F. de la Vega, I. Zergioti, Appl. Surf. Sci. 465, 136 (2019)

41. M. Makrygianni, I. Kalpyris, C. Boutopoulos, I. Zergioti, Appl. Surf. Sci. 297, 40 (2014)

42. C. Boutopoulos, I. Kalpyris, E. Serpetzoglou, I. Zergioti, Microfluid. Nanofluidics 16, 493 (2014)

43. L. Yang, C. Y. Wang, X. C. Ni, Z. J. Wang, W. Jia, and L. Chai, Appl. Phys. Lett. 89, (2006).

44. L. Rapp, J. Ailuno, A.P. Alloncle, P. Delaporte, Opt. Express 19, 21563 (2011)

45. P. Sopeña, J. Arrese, S. González-Torres, J.M. Fernández-Pradas, A. Cirera, P. Serra, A.C.S. Appl, Mater. Interfaces 9, 29412 (2017)

46. R. Pohl, C. W. Visser, G. R. B. E. Römer, C. Sun, A. J. Huis In'T Veld, and D. Lohse, J. Laser Micro Nanoeng. 10:154 (2015).

47. J. Miksys, G. Arutnov, M. Giesbers, and G. Römer, in Proc. LPM2018 (2018), pp. 1-4.

48. http://www.pvnanocell.com/sicrys-p75db-1.html\#.

49. M. Duocastella, J. M. Fernández-Pradas, J. L. Morenza, and P. Serra, J. Appl. Phys. 106, (2009).

50. S. A. Mathews, R. C. Y. Auyeung, H. Kim, N. A. Charipar, and A. Piqué, J. Appl. Phys. 114, (2013).

51. X. Liu, D. Du, G. Mourou, IEEE J. Quantum Electron. 33, 1706 (1997)

Publisher's Note Springer Nature remains neutral with regard to jurisdictional claims in published maps and institutional affiliations. 